\title{
Teaching Corner: Adult Asthma in Malawi
}

\section{Coleman}

Dept of Medicine, Queen Elizabeth Central Hospital, Blantyre

Asthma affects millions of people worldwide. It is often considered to be benign and is therefore under-diagnosed and under-treated. In fact asthma can be a very serious condition with significant morbidity and mortality. Most of the deaths are in patients over the age of 45 and many deaths could have been prevented with prompt medical treatment and good long term care ${ }^{1}$. The exact incidence of asthma in Malawi is unknown but it is a common clinical diagnosis. Recognising and treating asthma appropriately will save lives.

\section{Definition}

Diagnosis of asthma is clinical. It requires a history of variable airways obstruction, with more than one of the following symptoms: wheeze, cough, chest tightness and breathlessness ${ }^{2}$. The symptoms are usually episodic, so between 'asthma attacks' a patient may be completely well and have normal respiratory function.

\section{Pathogenesis}

Asthma is a disease that affects the small and medium airways in the lungs (bronchi). It is caused by airway hyper-responsiveness and immune activation leading to bronchoconstriction, inflammation and increased mucus production. These processes narrow the airways and cause symptoms of cough, chest tightness, wheeze and breathlessness.

The exact cause of asthma is unknown. It is thought to be due to a combination of genetic predisposition and allergen exposure. In some patients there is a clear family history of asthma, suggesting a stronger genetic component. We also know that patients with low birth weights and those born to smokers are more likely to develop asthma ${ }^{3}$.

In an asthmatic patient the airways are more sensitive to certain triggers. Once exposed to these triggers, a cascade of inflammation starts - excess autonomic activation leads to smooth muscle constriction; immune system activation mediated by immunogloubulin $\mathrm{E}(\mathrm{IgE})$ increases mast cell degranulation and release of inflammatory cytokines. This is associated with increased activation of eosinophils. Mucus production and inflammation of the bronchial wall lead to further airway narrowing.

\section{Diagnosis - Is it really asthma?}

Making a firm diagnosis of asthma is not as easy as many people think. There is no single diagnostic test, and your patient may appear in perfect health between attacks. The diagnosis is made clinically - the more positive elements there are the more likely the diagnosis. A careful history may elicit previous episodes of breathlessness and triggers associated with the onset of symptoms. Recurrent cough is common and a nocturnal cough may be the only reported symptom.

Many patients have been diagnosed with asthma as a child. It is important to review the history and treatment response in these patients to ensure the correct diagnosis.

A history of eczema or hay fever (atopy) increases the risk of asthma as does a family history of asthma or atopy (particularly maternal) ${ }^{4}$.
Examination findings can be unhelpful when a patient is well, but wheeze will help to confirm your diagnosis.

\section{Pointers towards a diagnosis of asthma}

1. Clinical suspicion of asthma

History of episodes of wheeze, recurrent breathlessness, cough and or chest tightness

Triggers for episodes of breathlessness

Well between episodes

\section{History suggestive of asthma}

Personal history of recurrent symptoms

History of childhood asthma

Improvement on treatment

Symptoms worse at night or early morning

Family history of asthma or atopy (asthma, eczema, allergic rhinitis)

\section{Examination findings}

\section{Normal}

Wheeze and reduced air entry during an attack

\section{Trial of treatment}

Clinical improvement on bronchodilators

\section{Investigations}

CXR, blood tests

Patients who have a history and examination suggestive of asthma, a trial of treatment is appropriate. They must be reviewed for clinical improvement to confirm the suspicion of asthma. If they do improve, then you can continue the asthma therapy with reasonable confidence. If patients do not respond to treatment, other diagnoses must be considered. Peak expiratory flow rates (PEFR) should be used to monitor the patient both before a trial of therapy and during it.

\section{Peak Expiratory Flow Rate (PEFR)}

A PEFR or 'peak flow' is a measurement of the fastest air flow during forced expiration. When the airways are narrowed by bronchoconstriction during an asthma attack, the airflow is reduced, so the PEFR falls. The PEFR may start to fall before the patient is aware of symptoms, so can be useful as an early warning of an asthma attack. Patients with asthma show more 'variability' in their PEFR measurements than non-asthmatic patients. This is due to small, often subclinical, variations in airway narrowing and bronchoconstriction. These variations also help in the diagnosis of asthma as this is a characteristic pattern. The PEFR is measured using a PEFR meter (Figure 1). This is simple to use but good patient technique is essential for reliable readings. The PEFR for each patient will depend on their height, age and sex, so each patient with asthma should know what their 'predicted' (calculated by age, height and sex) or 'best' (the PEFR they can achieve when well and asymptomatic) PEFR should be. 


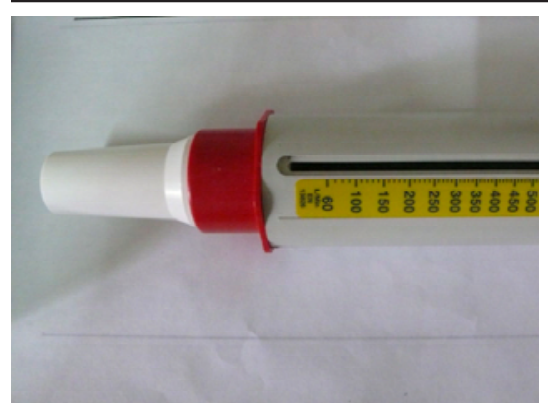

Figure 1. Peak Expiratory Flow Rate (PEFR) meter

\section{Other investigations}

Further investigations are only necessary if there is still diagnostic uncertainty. A chest X-ray may show the typical hyper-inflation with flattening of the diaphragms and elongation of the mediastinal shadow associated with asthma. Significant interstitial shadowing, lymphadenopathy or scarring and loss of volume all suggest alternative diagnoses.

Blood tests can be helpful but these tests are not widely available in Malawi, and are not necessary in the clinical diagnosis of asthma.

\section{Spirometry}

It is possible to measure flow through the airways using a simple piece of equipment called a spirometer. We measure the amount the patient is able to breathe out in one second (Forced Expiratory Volume in 1 second, or FEV1). We can also measure the total amount a patient is able to breathe out (Forced Expiratory Volume, FVC). The actual values vary with age, height and sex. The ratio of the FEV1 to FVC gives us a measure of airway obstruction which reflects airway narrowing. As airway narrowing (bronchoconstriction) is variable in asthma, the FEV1/FVC ratio will also vary. When a patient with asthma is well and has no symptoms, the FEV1/FVC ratio will be normal. During an asthma attack, or when the patient is less well controlled, the ratio may fall, showing obstruction caused by bronchocontriction.

This gives us another helpful test in asthma - if a reduced FEV1/FVC ratio is found (obstructive pattern) then you can try to reverse the obstruction by giving the patient a bronchodilator such as inhaled salbutamol. This reduces the bronchoconstriction, improving air flow out of the lungs, and the FEV1/FVC ratio will normalise. The obstruction is gone. This demonstrates 'reversibility' which is a key feature of asthma and explains how the patient's symptoms come and go. Other diseases that cause airway narrowing and an obstructive pattern on spirometry (such as Chronic Obstructive Lung Disease, COPD), are not readily reversible, so even with salbutamol the FEV1/FVC ration will remain the reduced.

\section{Triggers}

Many patients with asthma are able to identify 'triggers' that precipitate an attack. This may be tree or grass pollen, dust, road pollution, exercise or even a change in the weather. Identifying these triggers helps people to predict when they will feel symptomatic, and therefore to either avoid the trigger completely (cigarette smoke) or to increase their medication accordingly (weather).

However we also know that certain oral medications worsen asthma. Some of these, such as B adrenergic receptor antagonists (B blockers) cause bronchial smooth muscle constriction so narrowing the airway and worsening symptoms of asthma. Others, such as aspirin and non-steroidal antiinflammatory drugs (NSAIDS) act by blocking the COX1 enzyme and worsen asthmatic symptoms ${ }^{5}$. However not all patients with asthma will have hypersensitivity to NSAIDS and aspirin ${ }^{6}$.

\section{Management of asthma in Malawi \\ Bronchodilation}

Bronchodiation is essential to control of acute episodes of asthma and salbutamol is the most commonly available bronchodilator in Malawi. It is a short acting beta-agonist, which works on beta adrenergic receptors in the lung causing smooth muscle relaxation and bronchodilation. Salbutamol also acts on the cardiac muscle where it has a stimulatory effect, causing tachycardia.

Salbutamol can be given as an inhaled preparation, as a nebuliser, orally or, in extreme circumstances, intravenously. The method of delivery determines the rate of onset of action and likely side effects. Oral salbutamol has a slower rate of onset - it has to be absorbed by the gut, but has a more prolonged action when compared to inhaled salbutamol ${ }^{7}$. However the side effects from oral salbutamol are often more pronounced (palpitations and tremor) and the slow onset of action limits its use in acute settings ${ }^{8}$.

Inhaled salbutamol has a direct effect on the smooth muscle of the bronchi, with only a small proportion absorbed into the systemic circulation. It is possible to give high doses of inhaled salbutamol before the side effects become problematic. However this only works if the inhaler is used properly. Many patients find using an inhaler very difficult even after repeated practice.

To sidestep the issue of inhaler technique, patients using a salbutamol inhaler should be taught to use a spacer device. This allows the patient to inhale the salbutamol with little need for co-ordination. They simply dispense the inhaler into one end of the 'spacer' and breathe normally from the other. It is possible to buy a spacer device, but it is also possible to make one yourself from a plastic water bottle (Figure 2)

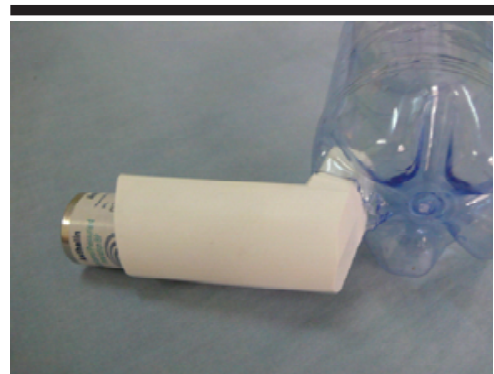

Figure 2. 'Spacer' made from a water or Sobo bottle

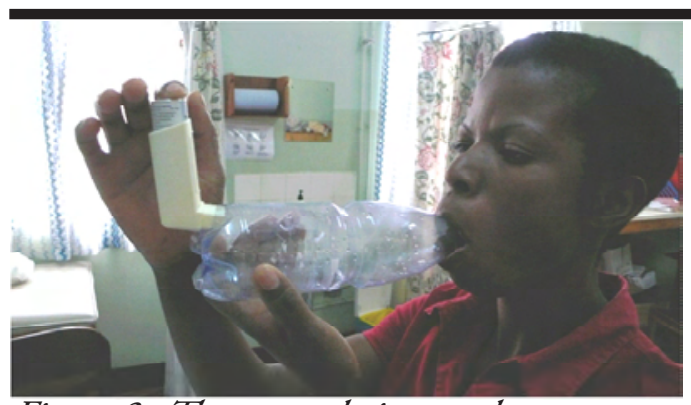

Figure 3. The spacer being used 
In the emergency setting, nebulised salbutamol is the most effective method of drug delivery. It does not require any co-ordination from the patient, and has a very rapid onset. The nebulisers should be repeated every 15 minutes until the patient improves. If a nebuliser is not available, inhaled salbutamol via a spacer is a good alternative.

Alternative bronchodilators include anticholinergic drugs, such as inhaled or nebulised ipratropium bromide. This is rarely available in Malawi. Adrenaline can also be used as a nebuliser if salbutamol is not available.

\section{Steroids}

All patients seen with an acute exacerbation of asthma need a short course of steroids. Steroids have a slow onset of action (4-6 hours, regardless of route of administration) but have an essential role in reducing the inflammation in the airways. Five days is normally appropriate. If your patient has been taking steroids for more than 10-14 days you should consider a tapering dose. Equally, all patients with regular symptoms of asthma - those who attend hospital for nebulisers, or use their inhalers more than 2-3 times a week, should be on a steroid inhaler. Steroid inhalers can (and should) be given via a spacer device.

\section{Other medications for asthma}

Magnesium sulphate is another drug used in acute asthma. The mechanism is unclear, but it is thought to stabilise mast cells and therefore reduce the ongoing inflammatory response.

Aminophylline is commonly used both in oral and intravenous forms. It is a methyxanthine (like caffeine) and has two main effects - it acts as an anti-inflammatory and also stimulates the central nervous system. Used regularly in oral form it can help to control asthma symptoms. It is used in the emergency setting for people with severe exacerbations. Patients who take oral aminophylline do not need to be given an intravenous loading dose.

\section{Complications of asthma - common pitfalls}

When a patient is deteriorating or fails to improve on treatment, there are several things that need to be considered. Most commonly the patient has simply not received enough bronchodilation therapy. However there are some other common complications that need to be considered.

Exhaustion is a serious consequence of a prolonged asthma attack - recognised by poor respiratory effort, reduced alertness and a falling respiratory rate. These patients need urgent anaesthetic review. Another cause of acute deterioration in an asthmatic is pneumothorax. This is a reversible life threatening event, and can be easily missed if you are not alert to the risk. Mucus plugging of the bronchi can cause acute deterioration - keeping your patient well hydrated, and encouraging her to cough will help. Chest physiotherapy is of great benefit if available. Infection is another common cause of either prolonged exacerbation or deterioration. Not all patients with acute asthma need antibiotics, but this should be considered in those with fever or purulent sputum.

\section{Other problems}

Patients who have not responded to treatment should be reassessed for alternative diagnoses. Diseases that can mimic asthma include chronic obstructive pulmonary disease, congestive cardiac failure (with a 'cardiac wheeze') and sarcoidosis (chronic cough and shortness of breath). Unilateral or localised wheeze should always raise the possibility of a focal obstructive lesion such as a foreign body or tumour. Acute allergy and anaphylaxis also cause acute bronchoconstriction, but do not warrant long-term bronchodilators. A thorough history and examination will help to differentiate between these conditions.

\section{Special cases of asthma}

\section{Chronic asthma}

Patients whose asthma has been well controlled over the years may remain totally asymptomatic between attacks. However if a patient's asthma has been undertreated (often either because of late diagnosis or poor adherence) they may develop chronic symptoms of gradually progressive shortness of breath, recurrent exacerbations and examination findings of hyperexpansion with reduced chest wall movement. This is due to chronic inflammation in the airways, with subsequent airways remodelling and irreversible narrowing. These patients may still have 'asthma attacks' but may remain short of breath or wheezy between episodes.

\section{Asthma in Pregnancy}

Asthma often worsens in pregnancy. Patients suffer from more frequent and more severe attacks. Pregnant women with asthma should continue on all their normal medication and should have their treatment escalated as needed?

Asthma is a common and highly variable chronic disease. It can be fatal, but usually responds well to prompt treatment. Careful out patient management is needed to prevent long term breathlessness and reduce emergency admissions.

\section{References}

\section{Braman S,The Global Burden of Asthma. Chest 2006;130;4S-12S}

2. British Guideline on Management of Asthma. British Thoracic Society and Scottish Intercollegiate Guidelines Network (SIGN)101. May 2008, revised 2009.

3. Sears M, Holdaway M, Flannery E, Herbison G, Silva A, Parental and neonatal risk factors for atopy, airway hyper-responsiveness, and asthma. Archives of Disease in Childhood 1996;75:392-398

4. Robert $\mathrm{J}$,Long-Term Risk Factors for Developing Asthma and Allergic Rhinitis: A 23-Year Follow-Up Study of College Students Settipane, Hagy G, Settipane G, Allergy and Asthma Proceedings, Volume 15, Number 1, January-February 1994 , pp. 21-25(5)

5. Szcezeklik A, Aspirin-induced asthma: advances in pathogenesis, diagnosis, and management. Stevenson D, Filter your current search Department of Medicine, Jagellonian University School of Medicine, Krakow, Poland. The Journal of Allergy and Clinical Immunologyfilter your current search [2003, 111(5):913-21; quiz 922]

6. Kasper L, Sladek K, Duplaga M, Prevalence of asthma with aspirin hypersensitivity in the adult population of Poland. Allergy 2003: 58: 1064-1066

7. Grimwood K, Johnson-Barrett J, Taylor B, Salbutamol: tablets, inhalational powder, or nebuliser? British Medical Journal 282: 105106, 1981.

8. S.Bhatia, H. Davies, Evaluation of Tolerance after continuous and prolonged oral administration of salbutamol to asthmatic patients. Br. J. clin. Pharmac. (1975), 2, 463-466

9. National Asthma Education and Prevention Program. Managing asthma during pregnancy. Recommendations for pharmacological treatment. Update 2004. http://www.nhlbi.nih.gov/health/prof/lung/ 